\title{
Acute and Chronic Renal Diseases Associated with Different Drugs in Children
}

\author{
Sergey Postnikov, Anna Gratzhianskaya, Marya Kostyleva, Aleksey Ermilin \\ Chair of Clinical Pharmacology, Pirogov Russian National Research Medical University, Moscow, Russian Federation
}

Email address

Kostyleva_m_n@rdkb.ru (S. Postnikov), annagrats@rambler.ru (A. Gratzhianskaya), Kostyleva_m_n@rdkb.ru (M. Kostyleva), elixin@list.ru (A. Ermilin)

\section{To cite this article:}

Sergey Postnikov, Anna Gratzhianskaya, Marya Kostyleva, Aleksey Ermilin. Acute and Chronic Renal Diseases Associated with Different Drugs in Children. American Journal of Pediatrics. Vol. 4, No. 3, 2018, pp. 61-68. doi: 10.11648/j.ajp.20180403.14

Received: June 28, 2018; Accepted: August 19, 2018; Published: September 19, 2018

\begin{abstract}
Drugs are common cause of renal damage - approximately $20 \%$ of out-patient and in-patient cases of acute renal failure are caused by drugs. The main reasons for this situation are the following: rapid growth of pharmaceutical market and therefore increased amount of drugs with known nephrotoxicity, aging of the population that is accompanied by the accumulation of such diseases like diabetes mellitus (DM), heart failure (HF), arterial hypertension (AH) following by the obligatory polypharmacy. On the other hand the achievements of contemporary medical science contribute to survivals of significant quantity of premature neonates with low and extremely low body weight. Thus the different types of pathological conditions like bronchopulmonary dysplasia (BPD), respiratory distress syndrome (RDS), sepsis, pneumonia, necrotic enterocolitis corresponding to such category of patients require aggressive treatment with off-label use. All factors listed above contribute to drug overload in the mentioned age-groups including drugs excreted by kidneys with possible damage of different renal structures and functions. The primary preventive measures could be the following: avoidance of the combination of drugs with known nephrotoxic effect, sufficient hydration and assessment of renal functioning using current biomarkers measuring before and in the progress of the treatment with potential nephrotoxic effect. It could be recommended the decreasing dose or interruption of the suspected drug in accordance with the deviation of parameters comparing to the baseline.
\end{abstract}

Keywords: Drug Nephrotoxicity, Mechanism, Damage of the Separate Nephron Structures, Risk Factors, Prevention

\section{Introduction}

Drug-induced renal diseases (DIRD) -damages separate renal structures with different severity and consequences that is caused by the pharmacological treatment.

In 1973 it was known [1] that no less than $10 \%$ of renal diseases are caused by drugs. It's obviously supposed that currently this percentage has to be significantly higher due to pharmacological market growth and prevalence of pharmacological treatment. In 2008 in accordance with the data [2] nearly $20 \%$ cases of acute renal failure are caused by drugs in out-patient and in-patient treatment periods.

However in contrast to hospital practice where the renal damage is almost iatrogenic, in out-patient practice the main reason for nephropathy in children of early age (together with the medical fault) is accidental intoxication (32.7\%) in particular with vitamins in accordance with the data [3]. In adolescents of 12-17 age old the predominant reason for nephrotoxicity is intentional poisoning (mainly by psychoactive drugs) with suicide intention (30.7\%), then alcohol intake (11.5\%) and drug abuse (19.3\%). In addition to listed above reasons the etiology of nephrotoxicity in children also includes the use of the following drugs: metamizole and non-steroid antiinflammatory drugs (NSAID) - acetylsalicylic acid, ketorolac, nimesulide, cytostatics, antibiotics (gentamicine), mix of drugs, cholinergic antagonists, iron drugs, enalapril. Predominantly the types of manifestations of renal damage are tubulopathy, tubular interstitial nephritis and nephrotic syndrome. Acute renal failure (ARF) occurs in $50.7 \%$ cases of toxic nephropathy caused by intoxication more often in adolescents that is followed by chronic renal disease in $39.5 \%$ [3].

In elderly patients DIRD develops in up to $66 \%$ [4]. The key role for its development plays the phenomenon of disease accumulation and polypharmacy related to this. 
Despite of possible reversion of DIRD if the suspected drug is interrupted at the proper time the more efforts and time are required in order to avoid the persistence of the process in elderly population.

\section{Classification}

DIRD are mainly classified by its localization (structural classification) - injured glomerulus, injured tubules, interstitial tissue, vessels. There is no strict relationship between the drug and the location of the damage in kidney, it means that the same drug may cause disease in different parts of nephron.

Distinction of acute and chronic processes in the course of DIRD is rather artificial as soon as some acute forms of DIRD caused by drugs with known toxic effect on kidneys like NSAID, aminoglycosides (AG), amphotericin B, the calcineurin blockers (cyclosporine, tacrolimus) may be followed by transformation into chronic form.

Regarding mechanism of development of DIRD it could be resulted from the direct toxic action of the drug (normally it's dose-dependent process), allergic reaction (idiosyncratic process) - dose NOT dependent, hemodynamic disturbances and obstructive nephropathy with deposition of drug crystals in the tubules lumen.

In addition it's necessary to distinguish DIRD by different types of the development - with direct nephrotoxic effect and those which initially manifest outside of kidneys (indirect, extrarenal process).

\section{Risk Factors of Nephrotoxicity}

It has to be distinguished between risks related to the patient and those related to the drug characteristics.

\subsection{Risk Factors Related to Patient}

1 Opposite parameters of age-groups: children of early age having immature and seems to be easily injured structures and elderly patients having involutive structural changes.

However some investigators note that renal damage caused by antibiotics (AB) in particular $A G$ and vancomycin occurs rarely and takes less severe course in full-term newborns than in children of other agegroups and adults. It seems to be paradoxical phenomenon but may be explained by immature structure of kidneys and therefore less tubular capture of xenobiotics and its less sensitivity to nephrotoxins [5].

However in prematurely born children ( $<37$ weeks gestational age) in particular in neonates with very low body weight $(<1500 \mathrm{~g})$ and extremely low body weight $(<1000 \mathrm{~g})$ the consequences of treatment by nephrotoxic drugs could be very severe. This is explained by incomplete nephrogenesis and big amount of histologically abnormal glomerules in this category of children. Moreover the condition of kidneys in prematurely born children could be negatively influenced by therapy given during pregnancy steroids, NSAID, ACE inhibitors [6].

Apart from the morphological basics for development of nephrotoxicity in premature newborns significant role is played by aggressive pharmacological treatment targeted on such diseases like RDS, BPD, severe infectious diseases (pneumonia), sepsis, necrotic enterocolitis that are specific for this age group of patients. It's necessary to emphasize that the treatment is performed mainly (in 90\%) off-label [6].

2 Development of DIRD is more probable in case of acute or chronic renal disease including oxalate or hyperuricemic nephropathy, renal micro- or macroanomalies. However manifestation of the drug-induced disease is often unrecognized as it's considered as the exacerbation of the primary renal disease.

3 Renal blood flow is the highest among body organs corresponding to $25 \%$ of cardiac output that threatens nephrotoxicity caused by drugs excreted by kidneys and taken for a long period of time or in high dosage. In case of decreased renal perfusion (chronic heart failure, bilateral renal arteries stenosis, arterial hypertension, diabetes mellitus) or in case of hypovolemia (persistent vomiting, prolonged diarrhea, agressive diuresis) the probability of renal drug-induced damage is increased.

4 In addition high biological activity of cells in kidneys determines its increased vulnerability including druginduced damage

5 Hypoalbuminemia due to hepatic disease and resulted from high level of free fractions of nephrotoxins in plasma.

6 The toxic metabolite of paracetamol - Nacetylbenzoquinonimine $(\mathrm{N}-\mathrm{ABQ})$ is not neutralized in case of low enzymatic status of liver or kidneys (e.g. low level of glutathione).

7 Pregnancy. In accordance with data [7] 63\% of pregnant women are suffered from latent form of glomerulonephritis or pyelonephritis. But even healthy pregnant women due to hormonal changes have atonic renal pelvis and ureter (more often on the right) that leads to urostasis and urinary reflux and therefore drug accumulation in kidneys in case of treatment of such patients. Aggravation of ureter paresis could be caused by ampicillin in accordance with [1].

\subsection{Risk Factors Related to Treatment}

1 Obviously that increased renal blood flow make possible for nephrotoxic drugs to permeate freely into renal structure. But the characteristics of the drug have the great deal. So the reasons for acute renal failure (ARF) are AG and amphotericin B in $40-45 \%$ and $80 \%$ accordingly. Vancomicin that is excreted with the help of glomerular filtration like AG (unchanged state in 80$90 \%$ ) is the reason for ARF in 6-30\% (in average 18\%) but cyclosporine which has only $6 \%$ of renal clearance rate (mainly as metabolites and only in $0.1 \%$ unchanged state) is the reason for renal damage in $18 \%$ as well [ 8 , 
9].

But nephrotoxicity is not the same even within AG group. The chemical structure of such $\mathrm{AB}$ is organic base with cation groups and its quantity likely correlates with the degree of nephrotoxicity. The quantity of these groups is the lowest in amikacin and consequently it has the weakest ability to damage [1].

2 Drug dose. Dose-dependent nephrotoxicity is noticed for example for vitamin $\mathrm{C}$ ( $>1 \mathrm{~g}$ per day), tetracycline ( $>2 \mathrm{~g}$ per day), colistin ( $>12 \mathrm{mg}$ per $\mathrm{kg}$ per day), fungizone $(>1 \mathrm{mg} \backslash \mathrm{kg}$ per day) and methotrexate $(>1$ $\mathrm{g} / \mathrm{m}^{2}$ ). That is why the treatment by the latter is performed under plasma concentration control which has to be below 1 mcmole/ $[10,11]$.

3 Duration of the treatment or the repeated courses with short intervals followed by the long-term effect (timedependent).

One example of such pathology could be so-called analgetic nephropathy that is resulted from more than one year analgetics-antipyretics abuse: analgin, paracetamol, NSAID (not only voltaren but also indomethacin plays a role) often in combination with the caffeine and codeine (with potential addicting property). In USA not rare use of analgetic mix causes ARF as a result of acute papillary necrosis or acute interstitial nephritis approximately in $1 \%$ of cases with the chronic renal disease as an outcome (analgetic kidney).

The more long-term consequence of analgetic nephropathy could be cancer of renal pelvis and urinary bladder which are revealed not earlier than in 15 years of analgetics abuse (cumulative dose 1-3 kg). But the precise frequency of such consequences are not known as the majority of patients die of chronic renal insufficiency before $[1,9]$.

4 Combined use and negative interaction of several (two or more) drugs with nephrotoxic effect, e.g.: $A G$ and furosemide, $\mathrm{AG}$ and amphotericin $\mathrm{B}, \mathrm{AG}$ and $\beta$ lactams.

In the first combination furosemide blocks secretion of AG increasing its intrarenal concentration that could lead to necronephrosis in case of continuous use of this combination [12].

In the second combination there is the summation of the nephrotoxic effects of both drugs (initially each of them has significant effect - up to $45 \%$ in AG and up to $80 \%$ in amphotericin B) acting through the different mechanisms of renal damage. Main characteristic of AG is to permeate to lysosomal cell apparatus in the epithelium of proximal tubules that is followed by the disturbance of protein synthesis and mitochondrion function. Amphotericin has double mechanism of action - preglomerular vasoconstriction (spasm of afferent glomerular arterioles) with hemodynamics type of disturbances in renal function and direct injury of epithelial cells in distal tubules with decreased reabsorption and exhaustion of $\mathrm{Na}^{+}, \mathrm{K}^{+}, \mathrm{Mg}^{++}$.
Interaction between $A G$ and $\beta$-lactams is multidirectional and is determined by the consequence of its use. If penicillin or cephalosporin is started before AG $\beta$-lactam blocks reabsorption of $A G$ occupying the places of its absorption that is evidence of $\beta$-lactam protective effect. If $\beta$-lactam is started after AG it potentiates nephrotoxic effect of AG. However protective effect of $\beta$-lactam could be smoothed in case of renal failure $[8,13]$.

\section{Direct Drug-Induced Damage of Renal Structure (Acute and Chronic)}

It's impossible to describe all drugs with nephrotoxic effect in one article (there are tens) thus we consider mostly used drugs.

\subsection{Glomerulopathy}

Let's focus on three groups of drugs.

First group: aurum salts, penicillamine, forscarnet, interferon- $\alpha$.

Toxic lymphokines initiate glomerular damage caused by drugs mentioned above.

Second group: Angiotensin Converting Enzyme inhibitors (iACE), Angiotensin Receptors I type Blockers (ARB I), vasopressors, calcineurin blockers, NSAID.

These drugs deteriorate glomerular function acting through hemodynamic mechanisms - changing of tonus of afferent and efferent arterioles followed by decreasing of glomerular filtration rate (GFR): NSAIDs block renal prostaglandins with vasodilatation effect, vasopressors stimulate $\alpha_{1}$ adrenoreceptors of vessels, calcineurin inhibitors damage endothelium of glomerular arterioles with endothelin-I release.

Mechanisms of damage induced by combination of iACE and ARB I are more complicated. On the one hand these drugs decrease GFR (in 3-5 days after treatment start) causing reduction of intraglomerular pressure due to block of angiotensine II vasoconstrictive effect. This is positive effect in case of renal disorders with proteinuria (diabetic kidney, nephrotic syndrome) or in case of arterial hypertension when kidneys are the target organs. On the other hand in the conditions of decreased renal perfusion (bilateral renal arteries stenosis, decompensated heart failure, hypovolemia) treatment by $\mathrm{iACE}$ and $\mathrm{ARB}$ I decreases even greater glomerular pressure that could cause renal failure (RF).

Morphological changes in glomerules are minimal in such diseases: focal segmental hyalinosis, glomerulonephritis or membranous nephropathy.

Stop drug treatment leads to involution of disturbances but it takes months and even years especially in case of aurum drugs [8].

Third group: drugs in this group induce glomerular damage targeting vessels with the development of thrombotic microangiopathy (thrombocyte blood clots in microcirculation) using two mechanisms: immune-mediated reaction (quinine, clopidogrel, ticlopidine) or direct vascular 
toxic effect with dose-dependent correlation (cyclosporine, tacrolimus) [2].

In addition a-interferon and NSAID are known to contribute to focal segmental glomerular sclerosis [14].

\subsection{Tubulopathy}

Acute tubular necrosis (vitamin C, tetracycline, AG, bisphosphonates, cisplatin, iodine containing contrast agents).

As mentioned above vitamin $\mathrm{C}$ and tetracycline have dosedependent type of damage mechanism.

Current stable opinion [15] regarding dose-dependent tubular toxicity of $\mathrm{AG}$ is questioned taking into account pharmacodynamics of such $\mathrm{AB}$ that means concentrationdependent bactericidal action in case of $\mathrm{C}_{\max }$ (e.g. for gentamicin) in 10 or more times higher than minimal inhibitory concentration (MIC) for this infectious agent that is achieved by one introduction of daily dose. In such a case possible nephrotoxic damage (determined by excretion of $\beta_{2^{-}}$ microglobulin) is not found [16]. It seems to find its causality in short period of action of high concentrations and therefore in longer duration of washing period that does not allow AG to accumulate in damaged organs. Besides this accumulation kinetics of one of AG - amikacin - and its related nephrotoxic effect has paradoxical nature: amikacin accumulates in cortical layer of kidneys in certain low serum concentrations and does not accumulate in high concentrations [17]. Thus amikacin introduction just once daily makes reach $C_{\text {min }}$ (concentration in serum before next drug introduction) less than $2 \mathrm{mg} \backslash \mathrm{l}$ which is safe regarding ototoxic and nephrotoxic effects. Paradoxicality of this phenomenon could be explained by low saturation capability of tissues regarding $\mathrm{AG}$ that means reduction or even termination of AG entry into tissues upon achievement of determined level of concentration [18].

The most informative parameters of acute (as a rule reversal) damage of proximal tubules (target for the toxic effect of AG) are the following: appearance in urine Kim-1 (kidney injury molecule-1), CysC (cystatine C), IL-18 (interleukin-18), NGAL (neutrophil gelatinose-associated lipocain), b2 and a1 - microglobulins, enzymuria (increased level of a-glucosidase and a-alaninaminopeptidase) $[6,19$, 20]

However AG tends to cumulate into lysosomes from which AG eliminates in 30 times lower than from plasma. Therefore prolongation of AG treatment ( $>14$ days) or recurrent treatment courses (e.g. in mucoviscidosis) could be followed by exceeding of threshold level of accumulation, cell damage with necrosis and outcome into chronic renal disease [21, 22]. Thus AG nephrotoxic effect could be considered more likely time-dependent than dose-dependent.

Besides of recommended duration of treatment (10-14 days) the following measures could facilitate nephrotoxic effect of AG in accordance with [8]:

a. Use of calcium drugs (or calcium channels blockers) through competitive inhibition of calcium channels;

b. Use of antioxidants (vitamin E, selenium, probucol, deferoxamine) because AG contributes to formation of free forms of $\mathrm{O}_{2}$ and to release of iron from mitochondrion in cells of cortical layer;

c. Combined treatment by AG and $\beta$-lactams introducing $\beta$-lactams at first;

d. Protective characteristics of recommended dosing [23] for AG taking into account GFR (decreasing of GFR is one of delayed AG nephrotoxic effect).

\subsection{Contrast-Induced Nephropathy (CIN)}

Radiocontrast substances (RCS) are the third cause of ARF in hospitalized patients [2]. However its frequency could be varied depending on study design, type and dose of the contrast and risk factors among those the main one is decreasing of GFR $<60 \mathrm{ml} \backslash \mathrm{min} \backslash 1.73 \mathrm{~m}^{2}$ in particular in patients with diabetes mellitus.

Being excreted by glomerular filtration in kidneys and concentrated in tubules RCS perform direct epithelial toxic effect by reactive forms of $\mathrm{O}_{2}$. In addition to mentioned above the genesis of tubular toxicity is specified by ischemic damage of tubules due to acute vessel constriction [2] (hemodynamics type of disturbances) and by ability of RCS to change the flexible characteristics of erythrocytes such as changing of its shape passing the blood capillary flow with return of $\mathrm{O}_{2}$ to tissues (hypoxic type of disturbances) [24]. Such characteristic of RCS is taken into account choosing the drug with low-osmolal and iso-osmolal characteristics in the lowest dose (e.g. Visipaque ${ }^{\circledR}$ ).

The following prevention measurements are recommended:

1 General measurements: assessment of basic renal functions before investigation, correction of risk factors if possible, avoidance of other drug with nephrotoxic effect;

2 Supporting treatment: optimal hydration regimen (physiological solution $1 \mathrm{ml} \backslash \mathrm{kg}$ during 18-26 hours: 612 hours before and 4-12 hours after RCS introduction); introduction of euphyllin $200 \mathrm{mg}$ per $100 \mathrm{ml}$ physiological solution before RCS and acetylcysteine (drug with antioxidant activity) $1200 \mathrm{mg}$ (600 mg twice) one day before investigation and the day after investigation. In the latter case nephrotoxicity decreased more than 10 times $[2,13,15]$.

\subsection{Obstructive Nephropathy}

Tubular damage could be also caused by drugs which form crystals insoluble in urine precipitating usually in distal tubular and blocking the urine flow that causes interstitial reactivity. The list of such drugs includes $\mathrm{AB}$ (ampicilin, oral cephalosporins, ciprofloxacin) and sulfonamides, antiviral drugs (acyclovir, foscarnet, ganciclovir, indinavir), methotrexate, triamterene. The probability of development of crystal tubulopathy depends on dose and duration of treatment, combination of mentioned above drugs and $\mathrm{pH}$ of urine, this probability increases in case of hypovolemia, existing renal insufficiency and in premature newborns with 
low body weight. For example acetazolamide and topiramate using both as antiepileptic drugs make urine reaction more alkaline by suppression of carbonic anhydrase in proximal tubulars that increase calcium excretion with its deposition in tubulars and renal parenchyma. Sulfonamides make alternative (acid) urine reaction that increases its crystal generating ability $[2,6,15,25]$.

\subsection{Interstitial Tissue Damage}

\subsubsection{Acute Interstitial Nephritis}

Acute interstitial nephritis (AIN) is an acute inflammatory reaction with involvement of interstitial tissue and tubules followed sometimes by systemic symptoms (fever, urticarial, arthralgia, eosinophilia) and causing ARF in 3-15\% [15]. Frequency of AIN (morphologically confirmed) is $10-18.6 \%$ and in majority cases (93\%) is related to drugs especially those excreted by kidneys [26].

AIN - not dose-dependent reaction when drug combines with tubular antigen (haptenization) or is deposited in interstitial tissue induced immune reaction through likely Tlymphocytes because they are contained in interstitial tissue [15]. Drugs could form circulating immune complexes able to be deposited in interstitial tissue and tubular structures followed by immune reaction through activation of complement and chemotaxis [26].

List of drugs causing AIN is rather big but more frequently these are $\mathrm{AB}(30-49 \%)$ mostly $\beta$-lactams (penicillin, cephalosporin) and also AG, rifampicin, ciprofloxacin, clarithromycin [8, 27, 28].

AIN usually develops on $10^{\text {th }}-14^{\text {th }}$ day after treatment start but it could happen earlier in patients already sensitized. The process like this is spontaneously reversible after interruption of "offending" drug if damage duration is less than two weeks. If there is no improvement prednisolone (dose $0.5-1$ $\mathrm{mg} \backslash \mathrm{kg} \backslash$ day) is prescribed (after biopsy) for 3-4 weeks with following dose decrease. If steroids have no effect it's considered to use cytotoxic drugs - cyclosporine, cyclophosphamide, mycophenolate mofetil. Progression of the disease at the background of treatment is the indication for dialysis.

\subsubsection{Chronic Interstitial Nephritis (CIN)}

CIN has dual mechanism of development:

a) inhibitors of calcineurin (tacrolimus, sirolimus) act trough hemodynamics disturbances causing vasoconstriction of preglomerular vessels;

b) in case of paracetamol, aspirin, NSAIDs (including selective) treatment CIN (necrosis and fibrosis of medullar interstitial tissue) develops as a result of its long use ( $>2$ years) with high dosage ( $>1$ g\day) - cumulative effect $[2,9]$.

\subsection{Papillary Necrosis}

This is specific complication of treatment causing by infarct of one or more papillas. Papillary necrosis could develop in all cases when there is disturbances of blood supply in renal papillas - drug shock, vasa recta damage in case of analgetics abuse, inhibition of vasodilation effect of prostaglandins by non-steroids, rarely as sulfonamides allergic reaction, or while performing retrograde urography. It is supposed that NSAID metabolites could accumulate in papillas and at threshold level provoke lipids peroxidation with tissue damage. Acetaminophen damaging effect is performed by its epoxide (N-ABQ) if this toxic metabolite could not be deactivated by renal medullary glutathione $[1,9$, $15]$.

\section{Extrarenal Causes of Nephrotoxicity}

\subsection{Hemolytic Kidney}

More than hundred drugs could cause intravascular hemolysis. It is caused intratubular block due to binding of tubular protein Tamm-Horstall with free hemoglobin and development of acute tubular necrosis with ARF. Druginduced hemolysis is not dose-dependent and could be related to allergic reactions II and III types (cytotoxic and immunocomplex) or being pseudoallergic (vitamindependent and enzymopenic) (Table 1) [29, 30].

Table 1. Pseudoallergic causes of hemolysis.

\begin{tabular}{ll}
\hline Erythrocytice & Extraerythrocytic \\
\hline Deficit of glucose-6-phosphate dehydrogenase & $\begin{array}{l}\text { Deficit of hepatic glutathione which paracetamol toxic metabolite binds with. } \\
\text { Deficit of reduced glutathione }\end{array}$ \\
$\begin{array}{l}\text { Deficit of glutathione-dependent enzymes: glutathione-reductase and } \\
\text { glutathione-peroxidase }\end{array}$ & $\begin{array}{l}\text { Deficit of vitamin } \mathrm{B}_{2}-\text { activator of glutathione-reductase. } \\
\text { (glutathione precursor). }\end{array}$ \\
\hline
\end{tabular}

Drug-induced hemolytic uremic syndrome (HUS) is similar to hemolytic kidney by its clinical manifestations but is different by pathogenesis. HUS could be caused by use estrogen-containing contraceptives, antitumoral drugs (mitomycin, cisplatin, bleomycin), antiaggregants (ticlopidine, clopidogrel), immunosuppressive drugs ciclosporin, tacrolimus (post- transplantation HUS). It is considered that mechanism of drug-induced HUS is related to endothelial damage in vessels with formation of microthrombosis blocking the lumens of small arteries and capillaries in kidneys that leads to acute cortical necrosis with glomerular involvement [15].

\subsection{Shocking Kidney}

If BP decreases suddenly below 70-60 $\mathrm{Hg} \mathrm{mm}$ the renal blood flow diminishes at $85-90 \%$ and the severe disturbances of glomerular filtration ability and of tubular supply (tubular necrosis) appear. Ischemic disturbances are intensified by occurring compensated spasm of renal vessels. It's considered that $\beta$-lactams are the most frequent cause for drug shock [31]. 


\subsection{Hypovolemic Kidney}

The disturbances of renal perfusion could develop not only due to sudden pressure decrease but also as a result of massive bleeding (anticoagulants overdose), decrease of volume of circulating blood (VCB) caused by liquid loss due to long-term diarrhea, persistent vomiting, intensive sweating and inadequate compensation of water-electrolytic balance [15]. Double decrease of VCB induce $15-20 \%$ decrease of blood flow in kidneys [31].

\subsection{Hypokaliemic Kidney}

In case of hypokaliemic conditions (laxative abuse, longterm treatment by diuretics - mostly by loop diuretics and steroids) tubulopathy with dystrophic changes in epithelium of proximal tubules is developed, sometimes in severe cases it could be revealed necrosis with following calcification [1].

\subsection{Hepatorenal Syndrome}

Sometimes this syndrome develops in parallel damage of liver and kidneys - e.g. by NSAID. However more often its development is consistent manifesting by clinical hepatic signs that are followed by acute renal failure due to tubulopathy on $7^{\text {th }}-8$ th day. In severe cases (hepatic coma) hepatorenal syndrome could develop already on $2^{\text {nd }}-3^{\text {rd }}$ day. The causing drugs are aurum salts, levofloxacin, iron containing druds, phenobarbital, depakine (in children under 3 years), isoniazid. It's necessary to emphasize that this syndrome occurs more often in patients with already compromised kidneys [31].

\subsection{Rhabdomyolysis (Myolytic Kidney)}

Rhabdomyolysis is a syndrome with damage of skeletal muscles that manifests with lysis of myocites with myoglobin and creatinekinase release. Myoglobin could have direct nephrotoxic effect, cause obstruction of tubules or change GFR that leads to ARF.

Among 150 drugs and toxins causing rhabdomyolysis statins are most frequently mentioned but its adverse reaction in case of monotherapy is quite rare $-0.44: 10000$ patients-years of treatment (children and adolescents with primary hypercholesterolemia, elderly patients with atherosclerosis). The second not- honorable place in the development of myolysis is occupied by drugs with potential addiction - cocaine, heroin, methadone, methamphetamine. Long-term treatment by prednisolone or single high dose (pulse-therapy) could also induce rhabdomyolysis. Exceeding of propofol dose ( $>4 \mathrm{mg} \mathrm{kg})$ and prolongation of its infusion ( $>48$ hours) lead to manifestation of myolytic kidney [32]. In general drugs and alcohol is responsible approximately for $81 \%$ of rhabdomyolysis and in half of these cases ARF develops afterwards [2].

\subsection{Syndrome of Tumor Lysis}

This syndrome develops as a result of cytostatics use. The products of tumor lysis (uric acid and calcium phosphate) induce intratubular obstruction and acute tubular necrosis with development of ARF as a result of reduced hydration and urine alkalescence. The possibility for development of such kind of complications mostly depends on background condition of kidneys.

\subsection{Retroperitoneal Fibrosis (Ormond Disease)}

Such kind of complications (unilateral or bilateral) with external block of renal vessels and ureter followed by progressive renal failure of tubule-interstitial type could be revealed in case of regular intake of drugs for headache of vascular type (methysergide, ergotamine, dihydroergotamine) and antihypertensive drugs (hydralazine, methyldopa, pindolol, atenolol). In histological material of kidneys being in such condition there are interstitial sclerosis, tubular atrophy, hyalinosis of significant part of glomerular $[1,15]$.

\section{False Nephrotoxicity}

Appearance of new biomarkers of drug-induced nephrotoxicity (IL-18, KIM-1, NGAL, CYSc) makes possible to distinguish situations when urea and creatinine levels increase does not related to renal damage:

1 Trimethoprim, cimetidine - inhibit tubular secretion of creatinine in competitive manner following by increase of its serum concentration.

2 Steroids, tetracycline - are associated with catabolic reaction and increasing of urea level.

3 Ascorbic acid, cefoxitin, cefalotin, flucytosine, levodopa, methyldopa taken by patients could cause false positive result for creatinine in laboratory [15].

\section{Evidences of Drug-Induced Causality for Renal Damage}

\subsection{The Causal Relationship with the Drug Is Likely to Be Possible}

1 Overdose (intentional or accidental) of the drug with known nephrotoxic effect. In some cases the causal relationship with the drug is confirmed by the evidence of increased concentration of the drug in blood and (or) in urine.

2 Stop intake of the suspected drug or the decreased dosage lead to the improvement of the renal functioning. However it's important to take into account that the renal functioning could continuously decline after the drug interruption for example in case of $A G$ as it could be accumulated in kidneys.

3 Return of the renal failure after recurrent suspected drug intake (as a rule during first 4 days).

4 Antibodies to the drug or its metabolite are revealed.

5 DIN develops more often in patients having risk factors [30].

\subsection{Drug Causality Could Be Excluded}

1 If the drug intake is started after development of ARF or if the drug intake is interrupted more than 48 hours before ARF. 
2 If the other causes for ARF are revealed in that case.

3 If the improvement of the renal functioning is observed while the treatment is maintained with the drug in the same dosage rate.

\section{Prevention}

Hereafter some measurements for DIN prevention are described in general [2, 30]:

1 Providing the information to the patient (parents) regarding possible negative renal adverse reactions of the performed treatment and necessity for application to the doctor in case of its occurring.

2 Correction of the some risk factors for nephrotoxicity:

a. Effective drugs have to be used likely without nephrotoxic effect;

b. Combination of drugs with nephrotoxic effects has to be avoided;

c. Supporting measures for the hydration maintenance in order to avoid renal hypoperfusion and increased nephrotoxic effect related to drugs that modify the renal blood flow (ARB I, iAEC and NSAID) or facilitate the obstructive tubulopathy (methotrexate).

3 Assessment of the basic renal functions before treatment initiation by drug with potential nephrotoxic effect.

4 Monitoring of renal functions in dynamics by measuring of biomarkers of nephrotoxicity: IL-18, KIM-1, NGAL, CYSc, sCR.

5 Correction drug dose in accordance with the changing of renal functions or drug interruption.

\section{Conclusion}

Drug is a double-edged steel that means that together with the required helping effect it could have negative damaging effect (and not rarely) on any organ. That's completely correct for drug-induced nephrotoxicity. Kidneys are one of the most important organs (the other is liver) for elimination and drugs eliminated by kidneys and being chemically active substances able to interact with different renal structures disturbing its function. Many different circumstances contribute to mentioned above conditions: background changes in urinary tract (malformations, diseases in anamnesis), elderly patients, severe prematurity, combination of drug with nephrotoxic effect. It helps to avoid pathological influence on kidneys the following measures - firstly knowledge of the possibility of such pathology and secondly monitoring renal functions using biomarkers assessment in order to assume necessary and timely measures: decreasing of dose, drug interruption, dialysis.

«Drugs are useful poisons» (Paracelsus)

Always be precautious and preventive.

\section{Conflict of Interest}

The authors declare no competing interests related to this work, this topic, subject and content.

\section{References}

[1] G. Mazhdrakov "Drug-induced renal and urinary tract damage" in book of Mazhdrakov G. and Popkhristova P. "Drug-induced diseases" 1973 Sofia, Medicine and physical training, pp. 507-578.

[2] Cynthia A. Naughton «Drug-induced nephrotoxicity». Am. Fam. Physician 2008 Sep 15; 78 (6): 743-50.

[3] Chemodanova M. "Frequency and type of renal damage in poisonings in children and adolescents". Abstract of dissertation for a degree of PhD, St-Petersburg, 2012

[4] DHODI Dinesh K. et al. Drug-induced nephrotoxicity. International Journal of Basic \& Clinical Pharmacology, [S.1.], v. 3, n. 4, p. 591-597, jan. 2017. ISSN 2279-0780

[5] Lukianova E. "Nephrotoxicity of antibiotics in neonates". Good clinical practice. 2002, N2, pp. 53-62.

[6] Girardi, A. M., Raschi, E., Galletti, S., Poluzzi, E., Faldella, G., Allegaert, K., \& Ponti, F. D. Drug-Induced Renal Damage in Preterm Neonates: State of the Art and Methods for Early Detection. Drug safety (2015). 38: 535

[7] Manual on obstetrics, gynecology and perinatology edited by academician of Russian Academy of Medical Science G. Savelieva. Medical information agency. Moscow, 2006, p. 716.

[8] Pannu Neesh, MD, SM, FRCP; Mitra K. Nadim, MD. «An overview of drug-induced acute kidney injury». $\mathrm{http}: / /$ www.area-c54. It/public/overview of drug-induced acute kidney injury.

[9] Stamatakis Mary K. «Chronic kidney diseases». В кн. James E. Tisdale and Douglas A. Miller Drug-induced diseases. Second edition. Bethesda, 2010 Chapter 44, p. 872-84.

[10] Clinical pharmacology by Gudman and Gylman. Practice. Moscow, 2006. Chapter 52 Anticancer drugs II Antimetabolites. Folic acid antagonists, pp. 10079-83.

[11] Fleury, Mapi, Caroline Fonzo-Christe, Charline Normand and Pascal Bonnabry. "Confusion between Two Amphotericin B Formulations Leading to a Paediatric Rehospitalisation.” Drug safety - case reports (2016).

[12] Clinical pharmacology edited by V. Kukes. Moscow, 1991. Publishing by Moscow Medical Academy, p. 442.

[13] Pai Amy Barton and L. Mason Darius «Acute kidney injury». В кн. James E. Tisdale and Douglas A. Miller Drug-induced diseases. Second edition Bethesda, 2010 Chapter 43, p. 85371.

[14] E. Petrosyan Focal segmental glomerulosclerosis - etiological, pathogenetic, clinical and morphological specifics. Pediatrics. 2007, 86 (3)

[15] Choudhury Devasmita and Ziuddin Ahmad «Drug-associated renal disfunction and injury». Nature clinical practice nephrology (2006) 2, 80-91.

[16] S. Semykin, S. Postnikov, S. Polikarpova et al. "Comparing efficacy and safety of single and double dosing of amikacin in patients with mucoviscidosis». Bulletin of pediatric pharmacology and threpsology" 2006, v. 3, \#6, pp. 42-47 
[17] E. Sorokina "Pharmacokinetics of aminoglycosides II in children of early age having acute pneumonia". Problems on maternity and childhood, 1987, 10, pp. 39-41

[18] Lacy M. K., Nicolace D. P., Nightingale C. H. «The pharmacodinamics of aminoglycosides». Clin. Inf. Dis. 1998; 27: 23-7.

[19] McWilliam S. J., Antoine D. J., Jorgensen A. L., Smyth R. L., Pirmohamed M. Urinary Biomarkers of AminoglycosideInduced Nephrotoxicity in Cystic Fibrosis: Kidney Injury Molecule-1 and Neutrophil Gelatinase-Associated Lipocalin (2018) Scientific Reports, 8 (1), art. no. 5094

[20] E. Savelieva, A. Vialkova, E. Kulagina, L. Kutchenko. Endocrinopathy and renal pathology in children. Russian bulletin if perinatology and pediatrics, 2016, \#4, p. 217.

[21] L. Strachunskyi, T. Reshedko, M. Edelshtein et al. "Comparing activity of cefepime, and other antibiotics against nosocomial infections in Russia" Clinical microbiology and antibacterial chemotherapy, 2003, v. 5, \#3, pp. 259-71

[22] Y. Belousov, S. Shatunov. "Antibacterial chemotherapy". Reference guide for doctors, Moscow, 2001. Aminoglycosides, pp. 152-73

[23] D. Gylbert, R. Mellering j., D. Eliopulos et al. "Antibacterial treatment by Jay Sanford". publishing by Granat. Moscow, 2013, p. 640.

[24] Y. Poliaev, A. Petrushin. "Modern radiopaque substances and risk of nephropathy development". Children hospital, 2009, \#4, pp. 37-42.
[25] Tasian Gregory E., Jemielita Thomas, Goldfarb David S., Copelovitch Lawrence, Gerber Jeffrey S., Wu Qufei, and Denburg Michelle R.. Oral Antibiotic Exposure and Kidney Stone Disease. JASN June 2018 29: 1731-1740

[26] Dyadyk A. I., Kugler T. E. Acute drug-induced tubulointerstitial nephritis. Almanac of Clinical Medicine. 2017; 45 (7): 586-598. (In Russ.) https://doi.org/10.18786/2072-0505-2017-45-7-586-598

[27] G. Ketova, O. Ilyicheva, Y. Korotkov. "Gentamicin: interstitial nephritis”. Drugs safety, 1998, \#3, pp. 15-16

[28] Myers R. P., Laughlin K. Mc, Hollomby D. J. «Acute interstitial nephritis due to omeprazol». Am.j.gastroenterology 2001, 96: 3428-31.

[29] S. Postnikov, M. Kostylieva, S. Rubanskyi. "Rare side-effect of paracetamol use in child of 8 years old". Children hospital, 2009, \#3 (37), pp. 22-23

[30] Acute renal failure of iatrogenic origin". Section 1. General information. Drug safety \#1, 2000, pp. 31-38

[31] G. Mazhdrakov, N. Popov. "Renal diseases". Medicine and physical training. Sofia 1969, p. 559

[32] S. Postnikov, M. Kostylieva, G. Brusov. Case of "Propofol infusion syndrome" in child of 10 years old $\backslash$ Safety and risk of pharmacotherapy, 2016. \#4, pp. 5-10 